\title{
Dual-Axis Hanle Magnetometer Based on Atomic Alignment with a Single Optical Access
}

\author{
Gwenael Le Gal, ${ }^{1,2}$ Gaëtan Lieb, ${ }^{1,2}$ François Beato, ${ }^{1,2}$ Thomas Jager, ${ }^{1,2}$ Hervé Gilles, ${ }^{3}$ and \\ Agustin Palacios-Laloy $\circledast^{1,2, *}$ \\ ${ }^{1}$ CEA-LETI, MINATEC Campus, 38054 Grenoble, France \\ ${ }^{2}$ Université Grenoble Alpes, 38000 Grenoble, France \\ ${ }^{3}$ Centre de Recherche sur les Ions, les Matériaux et la Photonique, ENSICAEN, CNRS, CEA/IRAMIS, \\ Université de Caen, 14050 Caen Cedex, France
}

(Received 14 June 2019; revised manuscript received 24 September 2019; published 4 December 2019)

\begin{abstract}
The usual Hanle-effect magnetometers allow the measurement of two components of a very-low magnetic field using three orthogonal light beams. Here we present a scheme based on atomic alignment that requires only a single optical access for both a pump beam and a probe beam with a small angle between them. The results of experimental tests of this configuration are in close agreement with the theoretical predictions. We show that measurements of the third component should be possible by instrumentation of a partial depolarization of the pump beam. Such compact architectures open interesting perspectives for magnetometer arrays for medical imaging.
\end{abstract}

DOI: 10.1103/PhysRevApplied.12.064010

\section{INTRODUCTION}

Optical pumping allows one to prepare a desired macroscopic spin-polarization state in an atomic gas. The perturbation of such a spin polarization by the local magnetic field translates into a change of the gas optical properties, which yields a measurement of this magnetic field. Optically pumped magnetometers relying on this principle have reached high sensitivities similar to those of superconducting quantum-interference devices, without requiring very low temperatures $[1,2]$. Such sensors open new perspectives for navigation [3], space exploration [4], studies of fundamental symmetries $[5,6]$, magnetic imaging of biological currents in cardiography $[7,8]$, fetal cardiography [9], encephalography [10], and myography [11].

The simplest kind of perturbation of a polarized atomic gas is the loss of polarization caused by a magnetic field of appropriate direction. Since the pump absorption is minimal in the fully polarized state, such a depolarization manifests itself by increased absorption. This magnetically induced absorption shows a resonant behavior around the null field: this is the so-called Hanle effect [12].

For magnetometry purposes, the addition of a second probe beam makes it possible to obtain a dispersive (i.e., odd-symmetric) dependence of the optical signals (either optical absorption or anisotropic-refractive-index effects [13]) on the magnetic field, allowing real-time measurement of one or two magnetic field components. Such Hanle

\footnotetext{
*agustin.palacioslaloy@cea.fr
}

magnetometers were used notably for instrumentation of the spin-exchange-relaxation-free effect [14], yielding the highest sensitivities obtained with optically pumped magnetometers [15]. In their most-usual configuration they use a circularly polarized light beam, which pumps the atoms toward an oriented state (i.e., a state with $\left\langle S_{k}\right\rangle \neq 0$ when the light propagation is along $\vec{k}$ ). A second probe beam, detuned from resonance, linearly polarized, and propagating orthogonally to the first one, probes the transverse orientation of the atoms because of the Faraday rotation: the rotation of the polarization plane witnessing an orientation longitudinal to the probe propagation [16]. In this setup, the polarization rotation is proportional to the component of the magnetic field orthogonal to both the pump propagation direction and the probe propagation direction, as shown in Fig. 1. The addition of another probe beam propagating orthogonally to the two first beams allows one to measure a second component of the magnetic field.

This configuration, however, requires two or three optical accesses to the gas cell, a requirement that hinders current efforts to miniaturize magnetometers and place them in dense arrays for medical imaging. For this reason most of the current work on magnetic imaging relies on parametric resonance magnetometers [9,17-20], where the addition of radio-frequency (rf) fields allows the measurement of two or three components of the magnetic field with only a single light beam. However, rf cross talk between magnetometers could result in imperfections, notably spurious changes in the measurement direction, which need to be specifically addressed. 

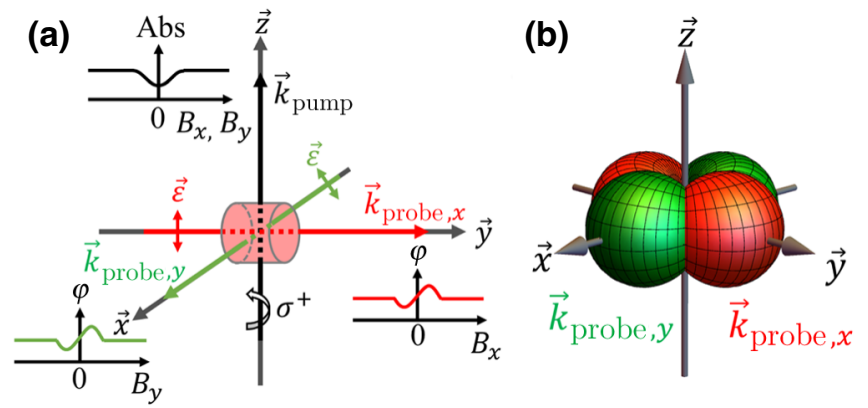

FIG. 1. (a) A dual-axis Hanle-effect magnetometer based on oriented spins and using two probes. Orientation is obtained by means of a circularly polarized pump beam. The linearly polarized probes undergo a polarization rotation $\varphi$ that shows dispersive dependence on the magnetic field components orthogonal both to the pump and the probe. The probe propagating along $\vec{x}(\vec{y})$ shown in green (red) allows the measurement of the $B_{y}\left(B_{x}\right)$ component. The pump-beam absorption (Abs) shows a even-symmetric dependence on the magnetic field. (b) Sensitivity to $B_{x}$ (in red) and $B_{y}$ (in green) as a function of the direction of the probe-beam propagation.

In this article, we propose another Hanle-magnetometer scheme with a reduced number of optical accesses. This scheme relies not on atomic orientation but on atomic alignment (i.e., states with $\left\langle 3 S_{e}^{2}-S^{2}\right\rangle \neq 0$ ). Atomic alignment can be created on any spin larger than $1 / 2$ by pumping with linearly polarized light, the direction of alignment $\vec{e}$ being that of the pump-light electric field $\vec{E}_{0}[21,22]$. Breschi and Weis [23] recently studied the Hanle effect on aligned atoms placed in an arbitrarily oriented magnetic field and with a single optical beam. Beato et al. [24] extended this theoretical analysis to all the components of atomic alignment, and found that they contain dispersive dependences on several magnetic field components. We complete here these studies by exploring theoretically multibeam configurations of the Hanle effect on aligned atoms (in Sec. II). From this analysis we deduce in Sec. III a particularly compact magnetometer scheme, and test it experimentally (Sec. IV). We present in Sec. V a further theoretical study of a three-axis Hanle magnetometer based on partially depolarized light.

\section{THEORETICAL MODEL OF THE HANLE EFFECT ON ALIGNED ATOMS}

For magnetometry purposes, it is convenient to decompose the density matrix $\hat{\rho}$ of an atomic state of total angular momentum $F$ on the magnetic multipole basis:

$$
\hat{\rho}=\sum_{k=0}^{2 F} \sum_{q=-k}^{k} m_{q}^{(k)} \hat{T}_{q}^{(k) \dagger}=\sum_{k=0}^{2 F} \sum_{q=-k}^{k} m_{q}^{(k)}(-1)^{q} \hat{T}_{-q}^{(k)},
$$

where $m_{q}^{(k)}=\operatorname{Tr}\left(\hat{\rho} \hat{T}_{q}^{(k)}\right)$ are the multipole moments of the atom, describing the atomic polarization of the ensemble, and $\hat{T}_{q}^{(k)}$ are the $2 k+1$ components of the irreducibletensor operators of order $k$. The order $k=0$ describes the total population of the metastable level, and the order $k=$ 1 describes the atomic orientation. We are interested here in $k=2$, which describes alignment, a tensor magnitude that can be obtained for any atomic level with $F>1 / 2$ (e.g. the $2^{3} S_{1}$ metastable level of ${ }^{4} \mathrm{He}$, with $F=1$ [25]). Its five components, which obey $m_{-q}^{(2)}=(-1)^{q}\left(m_{q}^{(2)}\right)^{*}$, can be represented by the column matrix $[23,24,26,27] M=$ $\left(m_{-2}^{(2)}, m_{-1}^{(2)}, m_{0}^{(2)}, m_{1}^{(2)}, m_{2}^{(2)}\right)^{t}$.

This alignment is subject to several concurrent processes: preparation of the gas state by optical pumping, relaxation, evolution under the magnetic field, and optical measurement. As shown elsewhere [23,28], if the pumping-light intensity is low enough, the dynamics of the system can be modeled following a three-step approach: (i) state preparation by optical pumping, (ii) evolution under a magnetic field and relaxation, and (iii) measurement of the system state.

In the case of metastable ${ }^{4} \mathrm{He}$, depopulation pumping is done with short-lived upper states (notably $2^{3} P_{0}$ ), and the excited-state population can thus be neglected. The equilibrium state in the absence of a magnetic field for linearly polarized pumping light along the quantization axis is given by

$$
M_{\mathrm{ss}}=m_{p}\left(0,0,6^{-1 / 2}, 0,0\right)^{t},
$$

where $m_{p}=\Gamma_{p} /\left(\Gamma_{p}+\Gamma_{e}\right)=\Gamma_{p} / \Gamma$, with $\Gamma_{e}$ the isotropic relaxation rate and $\Gamma_{p}$ the optical pumping rate.

In the presence of a small magnetic field $B_{0} \ll \Gamma / \gamma$, where $\gamma$ is the gyromagnetic ratio of the ${ }^{4} \mathrm{He} 2^{3} S_{1}$ metastable state, this equilibrium state is modified according to $[23,24]$

$$
\left(\frac{d}{d t}-\mathbb{H}(\vec{B})+\Gamma\right) M=\Gamma M_{\mathrm{ss}}
$$

where the matrix $\mathbb{H}(\vec{B})$ is given by

$$
-i \gamma\left(\begin{array}{ccccc}
-2 B_{z} & B_{-} & 0 & 0 & 0 \\
B_{+} & -B_{z} & \sqrt{\frac{3}{2}} B_{-} & 0 & 0 \\
0 & \sqrt{\frac{3}{2}} B_{+} & 0 & \sqrt{\frac{3}{2}} B_{-} & 0 \\
0 & 0 & \sqrt{\frac{3}{2}} B_{+} & B_{z} & B_{-} \\
0 & 0 & 0 & B_{+} & 2 B_{z}
\end{array}\right)
$$

where $B_{ \pm}=B_{x} \pm i B_{y}$ and $\vec{z}$ is taken as the quantization axis. 

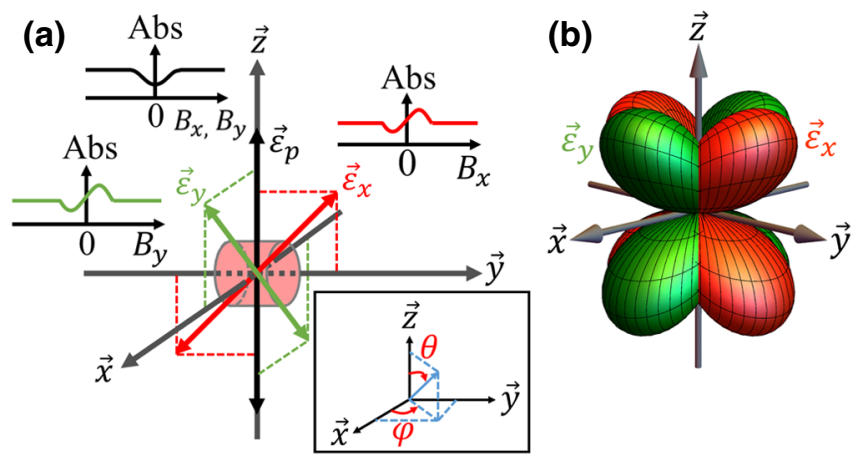

FIG. 2. (a) The dual-axis Hanle magnetometer based on atomic alignment. Two probe beams, with polarization $\overrightarrow{\varepsilon_{x}}$ and $\overrightarrow{\varepsilon_{y}}$, yield a dispersive dependence of the absorption (Abs) on two transverse magnetic field components $B_{x}$ and $B_{y}$. The inset defines the two spherical angles $\varphi$ and $\theta$ used in Sec. II. (b) Spherical plot of the sensitivity as a function of the direction of probe polarization in the architecture in (a) (red is for $B_{x}$ sensitivity and green is for $B_{y}$ sensitivity).

Solving Eq. (3) yields the solutions given in the Appendix, which at first order in the magnetic field are

$$
\begin{aligned}
& m_{2}^{(2)} / m_{p}=0+O\left(B_{i} B_{j}\right), \\
& m_{1}^{(2)} / m_{p}=-\frac{\gamma\left(i B_{x}+B_{y}\right)}{2 \Gamma}+O\left(B_{i} B_{j}\right), \\
& m_{0}^{(2)} / m_{p}=\frac{1}{\sqrt{6}}+O\left(B_{i} B_{j}\right),
\end{aligned}
$$

with $i, j \in\{x, y, z\}$.

The gas state can be monitored through its optical absorption, or its anisotropic refractive index [16]. The absorption coefficient can be separated into a constant contribution coming from $m_{0}^{(0)}$ and a variable part $\delta \kappa$. For a probe linearly polarized along $\vec{E}, \delta \kappa$ is proportional to $m_{0}^{(2)}$ in the frame where $\vec{E}$ is the quantization axis. Consequently, as shown elsewhere [23], there is no firstorder dependence on the magnetic field when the pump beam is used as a probe, because this dependence is evensymmetric. The idea is now to investigate the polarization directions that could display a dispersive dependence on each component of the magnetic field. $M$ changes of frame can be calculated with the Wigner $D$ matrix [29]. For the spherical angles $\varphi$ and $\theta$ shown in the inset in Fig. 2, we obtain at first order in magnetic field

$$
\begin{aligned}
\delta \kappa \propto \frac{m_{p}}{4 \sqrt{6}}[ & 1+3 \cos 2 \theta \\
& \left.+6\left(\frac{\gamma B_{y}}{\Gamma} \cos \varphi+\frac{\gamma B_{x}}{\Gamma} \sin \varphi\right) \sin 2 \theta\right] .
\end{aligned}
$$

The polarization direction exhibiting first-order dependences on the $B_{x}$ and $B_{y}$ components of the magnetic field are illustrated in Fig. 2 along with the corresponding sensitivity plot showing the sensitivity dependence on the probe-beam polarization direction. A probe beam polarized along the $\overrightarrow{\varepsilon_{x}}= \pm(\vec{z}+\vec{y}) / \sqrt{2}$ direction $(\theta=\pi / 4$ or $5 \pi / 4, \varphi=\pi / 2$ or $3 \pi / 2$ ) will allow one to probe the $B_{x}$ component with maximum sensitivity and a beam polarized along the $\overrightarrow{\varepsilon_{y}}= \pm(\vec{z}+\vec{x}) / \sqrt{2}$ direction $(\theta=\pi / 4$ or $5 \pi / 4, \varphi=0$ or $\pi$ ) will allow one to probe the $B_{y}$ component.

\section{TOWARD A DUAL-AXIS MAGNETOMETER ARCHITECTURE}

It should therefore be possible to build a dual-axis magnetometer with a pump beam linearly polarized along $\vec{z}$ and two probe beams linearly polarized along the $\overrightarrow{\varepsilon_{x}}$ and $\overrightarrow{\varepsilon_{y}}$ directions given in Sec. II. This means that the transverse $B_{x}$ and $B_{y}$ components can here be sensed by two linearly polarized beams, the relative directions of which form a $60^{\circ}$ angle (and not $90^{\circ}$, which once again reveals the rather counterintuitive behavior of the alignment). The polarizations along $\overrightarrow{\varepsilon_{x}}$ and $\overrightarrow{\varepsilon_{y}}$ can be addressed by a single beam propagating along $\overrightarrow{\varepsilon_{x}} \times \overrightarrow{\varepsilon_{y}}=\overrightarrow{k_{s}}$ as shown in Fig. 3(a). This allows one to access two components of the magnetic field from a single optical access. However, if both beams are sent onto the same photodiode, some way to separate the information has to be found. One possibility consists in performing two distinct amplitude modulations (i.e. with two different modulation frequencies on the two copropagating beams), so that the signals can be separated by filtering or demodulation. Contrarily to parametric resonance, which causes frequency modulation of the atomic multipole moments, and therefore reduces the signal-tonoise ratio [24], this amplitude modulation does not affect the signal-to-noise ratio. Moreover, the modulation also allows one to shift the Hanle signals to frequencies where the low-frequency laser noise is lower.

To achieve the most-compact architecture, the pump beam should ideally be sent through the same optical access as the probe beam. If the pump beam is sent along $\vec{k}_{s}$, then the pumping steady-state alignment cannot be along the $\vec{z}$ axis. We thus need another propagation direction, $\overrightarrow{k_{p}}$, orthogonal to $\vec{z}$ (pump polarization direction). Sending the probe beams and the pump beam through the same optical access is feasible if the angle between $\vec{k}_{s}$ and $\overrightarrow{k_{p}}$ can be made small enough. The minimization of this angle is obtained for $\overrightarrow{k_{p}}=-(\vec{x}+\vec{y}) / \sqrt{2}$, yielding $35.3^{\circ}$, which happens to be the complement to $90^{\circ}$ of the angle between the diagonal of the cube and one of its side. The resulting architecture is shown in Fig. 3(a); it comprises three beams: the pump beam propagating along $\overrightarrow{k_{p}}$ and 
linearly polarized along the $\vec{z}$ axis, and the two probe beams, both propagating along $\vec{k}_{S}$ and linearly polarized along $\overrightarrow{\varepsilon_{x}}$ and $\overrightarrow{\varepsilon_{y}}$. All those beams can be sent through the same optical access because of the small angle of $35.3^{\circ}$ between $\overrightarrow{k_{p}}$ and $\overrightarrow{k_{s}}$.

\section{EXPERIMENTAL IMPLEMENTATION OF A DUAL-AXIS MAGNETOMETER WITH ${ }^{4} \mathrm{He}$}

To check the predictions made in the previous section, we build the experimental setup as shown in Fig. 4(a), which reproduces the architecture shown in Fig. 3(a).

To have two copropagating beams with different polarization states, as described in Sec. III, we use a fiber assembly with two independent polarization-maintaining fibers assembled in a $\mathrm{V}$ groove. We are thus able to inject two collinear and optically independent beams with distinct polarization states separately and respectively amplitude modulated at $\omega_{1}$ and $\omega_{2}$. For practical reasons we measure the signals with the probe beam polarized along $\overrightarrow{\varepsilon_{x}}$ and $\overrightarrow{\varepsilon_{y}}$ sequentially.

A cylindrical cell of 10-mm diameter and 10-mm length filled with 9 -Torr ${ }^{4} \mathrm{He}$ is used. The metastable level is
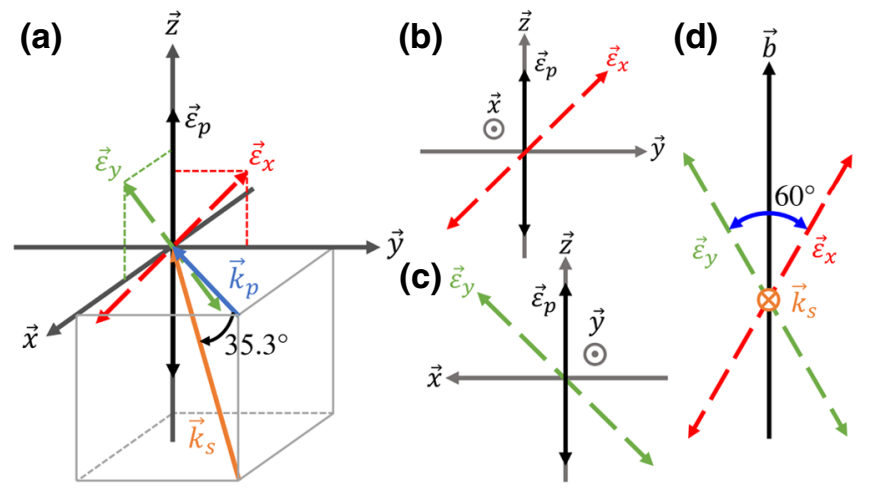

FIG. 3. Polarization directions in the dual-axis magnetometer based on atomic alignment. (a) Final scheme of the architecture. The pump polarization $\overrightarrow{\varepsilon_{p}}$ is along $\vec{z}$. The first-probe-beam polarization $\overrightarrow{\varepsilon_{x}}$ is in the $y-O-z$ plane at $45^{\circ}$ to both $\vec{y}$ and $\vec{z}$. The second-probe-beam polarization $\overrightarrow{\varepsilon_{y}}$ is in the $x-O-z$ plane at $45^{\circ}$ to both $\vec{x}$ and $\vec{z}$. The propagation direction that is common to both probe beams $\overrightarrow{k_{s}}$ is orthogonal to $\overrightarrow{\varepsilon_{x}}$ and $\overrightarrow{\varepsilon_{y}}$ and lies at $35.3^{\circ}$ beneath the $x-O-y$ plane. The pump-beam propagation direction $\overrightarrow{k_{p}}$ that minimizes the angle with $\overrightarrow{k_{s}}$ is in the $x-O-y$ plane at $45^{\circ}$ from $\vec{x}$ and $\vec{y}$. (b),(c) Projection onto the $y-O-z$ and $x-O-z$ planes, respectively, showing the orientation of $\overrightarrow{\varepsilon_{x}}$ and $\overrightarrow{\varepsilon_{y}}$ in those planes. (d) Front view in the $\overrightarrow{k_{s}}$ direction. $\vec{b}$ is the projection of $\vec{z}$ onto the plane transverse to $\vec{k}_{s}$ and passing through the origin of the frame. $\overrightarrow{\varepsilon_{x}}$ and $\overrightarrow{\varepsilon_{y}}$ form a $60^{\circ}$ angle, the bisector of which is $\vec{b}$. populated by use of a rf discharge at $22.8 \mathrm{MHz}$ absorbing $10 \mathrm{~mW}$ of electrical power. All the light beams are generated with a distributed-feedback laser diode from QD Laser tuned on the $D_{0}$ line of ${ }^{4} \mathrm{He}$. This beam is coupled in a polarization maintaining fiber and split into the pump and probe beams. The pump beam is linearly polarized along the $\vec{z}$ axis as shown in Fig. 3(a). The probe beam is modulated in amplitude at $65 \mathrm{kHz}$ with an acousto-optic modulator from AA Opto-electronic (MT110-IR20-FIO). As discussed in Sec. III, the two probe polarizations form a $60^{\circ}$ angle and, as shown in Fig. 3(c), this is the angle in the plane perpendicular to $\vec{k}_{s}$. The bisector $\vec{b}$ of this angle is the projection of the $\vec{z}$ axis on this plane. Thus, the polarization $\overrightarrow{\varepsilon_{x}}$ is set at $+30^{\circ}$ from $\vec{b}$ and the polarization $\overrightarrow{\varepsilon_{y}}$ is set at $-30^{\circ}$. The transmitted light is collected in a $200-\mu \mathrm{m}$-core multimode optical fiber and sent to an InGa-As photodetector (TTI TIA 525I). The signal is then demodulated with a lock-in amplifier (Stanford SR865A). The cell and beam collimators are placed at the center of a triaxial Helmholtz coil (Ferronato BH300HF-3-B) and the whole setup is placed inside a five-layer magnetic shield to operate in the low-field conditions required to observe the Hanle effect. The optical power experienced by the atoms is around $166 \mu \mathrm{W}$ for the pump and ranges from 0 to $34 \mu \mathrm{W}$ for the probe (corresponding to $211 \mu \mathrm{W} / \mathrm{cm}^{2}$ and 0 to $43 \mu \mathrm{W} / \mathrm{cm}^{2}$ respectively).

The sensitivity to each magnetic field direction, defined as the slope of the first-order dependence on the magnetic field components [Eq. (6)], is measured by generating magnetic field ramps of $\pm 300 \mathrm{nT}$ in the suitable direction with the triaxial coil. $\psi$, the angle between the $\vec{x}$ axis and the direction of the magnetic field in the $x-O-y$ plane, is shown in Fig. 4(b).

For each probe polarization $\left(\overrightarrow{\varepsilon_{x}}\right.$ and $\left.\overrightarrow{\varepsilon_{y}}\right)$, we measure the Hanle signals with the static field for different values of $\psi$ : they are shown as insets in Figs. 4(c) and 4(d). The slopes of the dispersive dependence are shown in Figs. 4(c) and 4(d).

These measurements show good agreement with the theoretical predictions. As shown in Fig. 4(c), we obtain a purely dispersive dependence when $\psi=0^{\circ}$ or $180^{\circ}$ (i.e., when the magnetic field is set along $\pm \vec{x}$, corresponding to the optimal slope predicted for $\overrightarrow{\varepsilon_{x}}$ ). A similar behavior shifted by $90^{\circ}$ in $\psi$ is observed for the $\overrightarrow{\varepsilon_{y}}$ signals [Fig. 4(d)]. The discrepancies in the amplitudes between the measurements for $\overrightarrow{\varepsilon_{x}}$ and $\overrightarrow{\varepsilon_{y}}$ arise from different optical coupling when the position of the probe collimator is changed. When the value of the slope decreases, the line shape becomes a sum of odd and even symmetric lorentzian signals. When the slope is zero, the shape is purely even-symmetric, showing only a second-order dependence on the given component of the magnetic field 


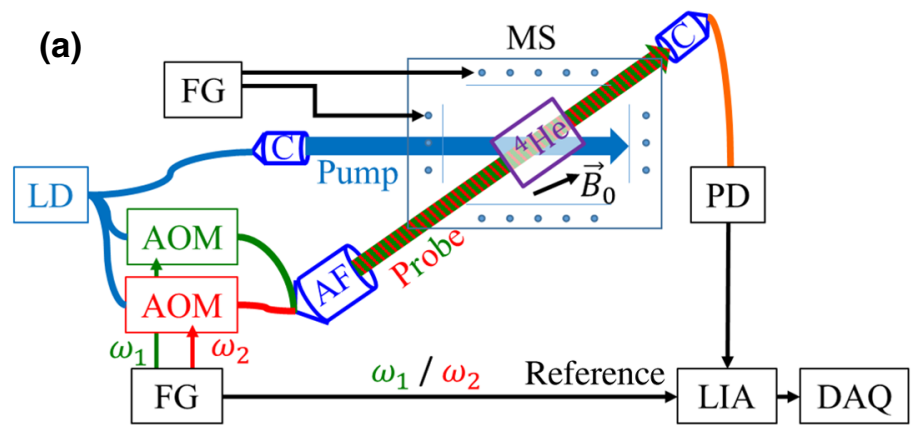

(b)

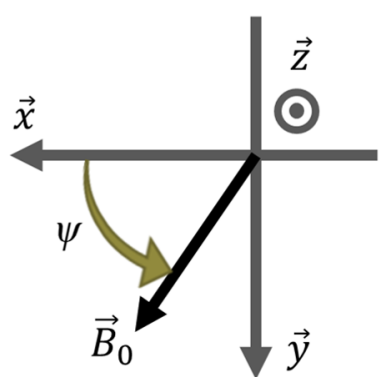

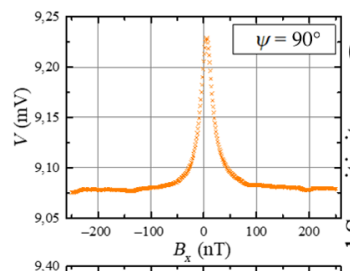

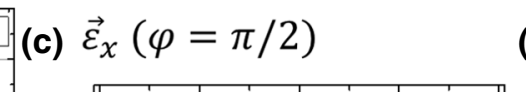

(d) $\vec{\varepsilon}_{y}(\varphi=0)$
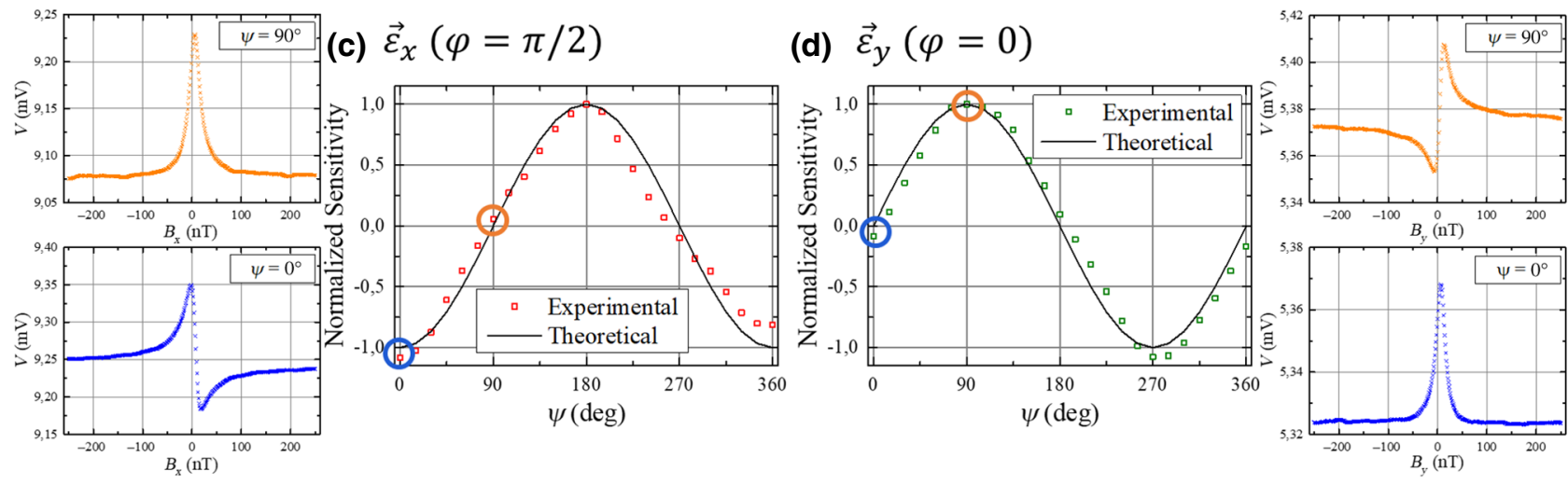

FIG. 4. (a) The experimental setup. (b) Definition of $\psi$, the angle between the $\vec{x}$ axis and the magnetic field $\overrightarrow{B_{0}}$ in the $x$-O-y plane. (c) $\overrightarrow{\varepsilon_{x}}$ sensitivity with the angle $\psi$. (d) $\overrightarrow{\varepsilon_{y}}$ sensitivity with the angle $\psi$. The sensitivity is normalized with respect to the maximum positive sensitivity obtained. The insets show the shape of the resonance signal for specific values of $\psi$. AF, assembled fiber; AOM, acousto-optic modulator; $\mathrm{C}$, collimator; DAQ, data acquisition; FG, function generator; LD, laser diode; LIA, lock-in amplifier; MS, magnetic shield; PD, photodiode.

( $B_{y}$ for $\overrightarrow{\varepsilon_{x}}$ and $B_{x}$ for $\overrightarrow{\varepsilon_{y}}$ ). These undesired deformations of the signals effectively hinder a proper measurement of an arbitrarily oriented magnetic field in an open-loop operation mode. However, since these deformations arise from magnetic field second-order terms and crossed terms in the signal, we can maintain optimal sensitivity and avoid measurement offsets by introducing a compensation field that cancels each component of the magnetic field measured by the magnetometer $\left(B_{x}\right.$ and $\left.B_{y}\right)$ in a closed-loop configuration [30]. If the magnetic field is low enough to observe the Hanle effect $\left(B_{0} \ll \Gamma / \gamma\right)$, the second-order terms in $B_{z}$ are expected to be negligible with respect to the first-order terms in $B_{x}$ and $B_{y}$ and will thus not hinder a proper measurement of these two components. The crossed terms are annihilated by the cancellation of the $B_{x}$ and $B_{y}$ components from the measurement.

In the experimental setup we operate, the intrinsic noise of the magnetometer is estimated to be limited by the photon noise. This noise can be evaluated like $B_{s n}$ in Ref. [8], but because of the amplitude modulation the average photon flux corresponds to the rms value of the photocurrent. This yields approximately $490 \mathrm{fT} / \sqrt{\mathrm{Hz}}$ for the $\overrightarrow{\varepsilon_{x}}$ polarization and $910 \mathrm{fT} / \sqrt{\mathrm{Hz}}$ for the $\overrightarrow{\varepsilon_{y}}$ polarization. These values could be increased with a better optical coupling (currently the optical coupling is $7.3 \%$ for the $\overrightarrow{\varepsilon_{x}}$ polarization and $3.9 \%$ for the $\overrightarrow{\varepsilon_{y}}$ polarization). We do not expect the sensitivity to be significantly degraded with both probe beams sent at the same time as long as the total optical power of the probe beams is low enough with respect to the optical power of the pump beam.

\section{TOWARD A THREE-AXIS MAGNETOMETER ARCHITECTURE}

The $B_{z}$ component of the magnetic field cannot be measured in the scheme presented in Sec. II, because it has no effect on the alignment longitudinal to the $\vec{z}$ axis. However, a measurement of this component could be possible if the atoms were aligned in a direction transverse to $\vec{z}$ (e.g., the propagation direction $\overrightarrow{k_{p}}$ ). Such steadystate alignment can be obtained in the very same optical scheme as described above if the pumping light is unpolarized [22,31]. Unpolarized light is a statistical mixture of all the possible polarizations; therefore, the steady-state alignment for a beam propagating along $\vec{z}$, taken as the quantization axis, is

$$
M_{\mathrm{ss}, \text { unpol }}=m_{p}\left[0,0,-(2 \sqrt{6})^{-1}, 0,0\right]^{t} .
$$




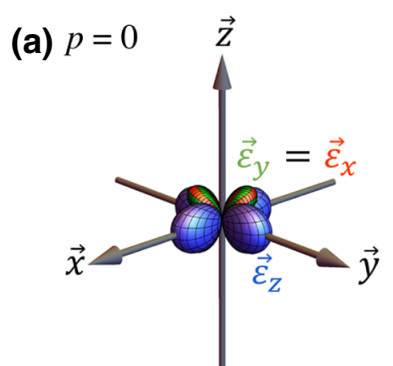

(b) $p=0.25$

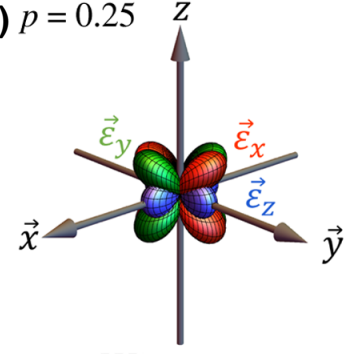

(c)

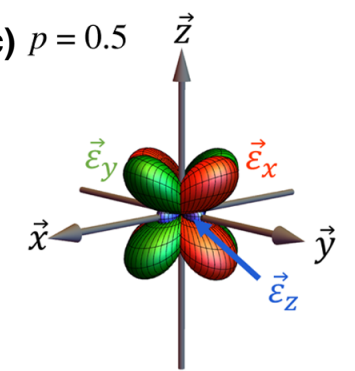

FIG. 5. Sensitivity as a function of the probe polarization for different degrees of polarization $p$ of the pump beam. The plots are shown on the same relative scale so that the sizes of the lobes are comparable. The plot in (e) is not on the same scale as the plot in Fig. 2(b).

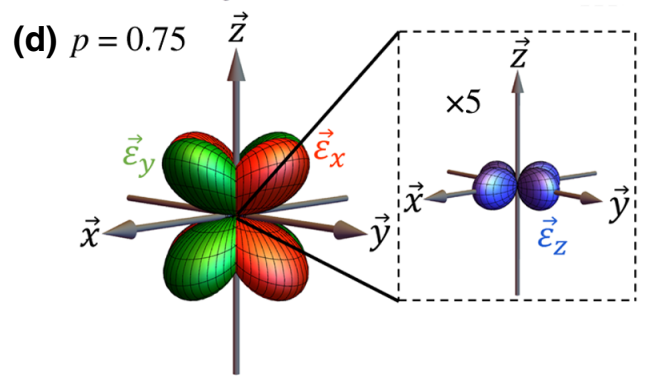

(e) $p=1$

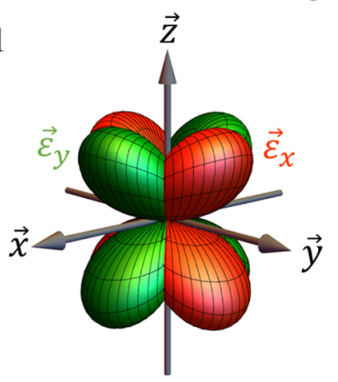

Thus, a partially linearly polarized pump beam propagating along $\overrightarrow{k_{p}}=-(\vec{x}+\vec{y}) / \sqrt{2}$ with polarization along $\vec{z}$ and a degree of polarization $p$ leads to nonzero transverse components in $M_{\mathrm{ss}}$, which is now written as

$$
M_{\mathrm{sS}}=m_{p}\left(\frac{-i(p-1)}{8}, 0, \frac{1+3 p}{4 \sqrt{6}}, 0, \frac{i(p-1)}{8}\right)^{t},
$$

with $\vec{z}$ being the quantization axis. The nonzero steadystate transverse multipole moments $m_{ \pm 2, \mathrm{ss}}^{(2)}= \pm \operatorname{im}_{p}(p-$ 1) $/ 8$ evolve with $B_{z}$. From Eqs. (8) and (3), one obtains the following expression for $\delta \kappa$ :

$$
\begin{aligned}
\delta \kappa \propto & \frac{1}{16 \sqrt{6}}\left\{(1+3 p)(1+3 \cos 2 \theta)-6(p-1) \sin ^{2} \theta \sin 2 \varphi+6\left[\frac{2 \gamma B_{z}(p-1)}{\Gamma} \cos 2 \varphi \sin ^{2} \theta\right.\right. \\
& \left.\left.+\left(\frac{\gamma\left[B_{y}(1+3 p)+B_{x}(p-1)\right]}{\Gamma} \cos \varphi+\frac{\gamma\left[B_{x}(1+3 p)+B_{y}(p-1)\right]}{\Gamma} \sin \varphi\right) \sin 2 \theta\right]\right\} .
\end{aligned}
$$

Because of such a partially depolarized linear pumping, one can obtain odd-symmetric dependences with all components of the magnetic field. Compared with Eq. (6), one can see in Eq. (9) that the $B_{z}$ component can be probed with maximum sensitivity using a probe beam polarized along the $\overrightarrow{\varepsilon_{z}}=\vec{x}$ direction or the $\vec{y}$ direction, whereas the $\varphi$ values are different from those extracted from Eq. (6) for the $B_{x}$ and $B_{y}$ components. Although they depend on the polarization degree $p$, one can still obtain odd-symmetric dependences for those components. When the pumping light is fully unpolarized $(p=0)$, the $\varphi$ value allowing maximum probing sensitivity is the same for $B_{x}$ and $B_{y}$ as a consequence of the symmetry of the corresponding directions with respect to the propagation direction of the pump beam $\overrightarrow{k_{p}}$.

As shown in Fig. 5, the degree of polarization also impacts the relative sensitivity of each axis. The Hanle effect is sensitive only to magnetic field components transverse to the pumping $[32,33]$. Therefore, when the alignment is purely transverse to $B_{z}(p=0)$, the $B_{z}$ sensitivity is maximal. When $p$ increases, the steady-state alignment becomes more longitudinal with respect to $B_{z}$, yielding a decrease of the sensitivity to zero when $p=1$.

On the other hand, the alignment always shows transverse components with respect to $B_{x}$ and $B_{y}$, which explains why we can probe them whatever the value of $p$. The sensitivity is, however, much lower when $p=0$ than when $p=1$ because the depolarization of the pumping light affects both the pumping efficiency and the resultant steady-state alignment direction. However, conversely to the $B_{z}$ case, the optimal $\varphi$ angle varies with $p$ for $B_{x}$ and $B_{y}$.

Nevertheless, building a three-axis magnetometer based on partially polarized light requires a third optical access to probe the last component ( $B_{z}$ in our case), thus making the architecture more cumbersome. 


\section{CONCLUSION}

We present a theoretical study of the Hanle effect on aligned atoms so as to develop a single-probe dual-axis magnetometer architecture. The results of experimental tests of the resulting configuration are in good agreement with theoretical predictions, and open the possibility for building a compact dual-axis Hanle-effect magnetometer.

We extend our theoretical analysis by proposing a three-axis Hanle-magnetometer architecture using partially depolarized light.

Besides its practical implications for magnetometry, this work highlights some counterintuitive features of the geometry of aligned states, such as the possibility of measuring two orthogonal magnetic field directions from a single optical access using two polarizations forming a $60^{\circ}$ angle. This opens interesting possibilities for the optical setup of Hanle magnetometers.

\section{ACKNOWLEDGMENTS}

We acknowledge the technical help of W. Fourcault for the optical setup of the experiment, cell filling by $F$. Alcouffe, and interesting discussions with E. Labyt, J.M. Léger, M. Le Prado, and F. Bertrand. G.L.G., G.L., and F.B. acknowledge CEA-LETI DSYS Ph.D. funding. This research work was supported by the French ANR via Carnot funding.

\section{APPENDIX: EXPRESSION FOR ALIGNMENT RESULTING FROM THE HANLE EFFECT WITH LINEARLY POLARIZED PUMPING}

The complete expression for the alignment $M$ solution of Eq. (3) is where $\Delta=2\left[\Gamma^{4}+5 \Gamma^{2} \gamma^{2}\left(B_{x}^{2}+B_{y}^{2}+B_{z}^{2}\right)+4 \gamma^{4}\left(B_{x}^{2}+B_{y}^{2}\right.\right.$ $\left.\left.+B_{z}^{2}\right)^{2}\right]$.

[1] V. Shah, G. Vasilakis, and M. V. Romalis, High Bandwidth Atomic Magnetometery with Continuous Quantum Nondemolition Measurements, Phys. Rev. Lett. 104, 013601 (2010).

[2] G. Vasilakis, H. Shen, K. Jensen, M. Balabas, D. Salart, B. Chen, and E. S. Polzik, Generation of a squeezed state of an oscillator by stroboscopic back-action-evading measurement, Nat. Phys. 11, 389 (2015).

[3] M. Nabighian, V. Grauch, R. Hansen, T. LaFehr, Y. Li, J. Peirce, J. Phillips, and M. Ruder, The historical development of the magnetic method in exploration, Geophysics 70, 33 (2005).

[4] N. Olsen, G. Hulot, and T. J. Sabaka, Measuring the Earth's magnetic field from space: Concepts of past, present and future missions, Space Sci. Rev. 155, 65 (2010).

[5] P. Knowles, G. Bison, N. Castagna, A. Hofer, A. Mtchedlishvili, A. Pazgalev, and A. Weis, Laser-driven Cs magnetometer arrays for magnetic field measurement and control, Nucl. Instrum. Methods Phys. Res., Sect. A 611, 306 (2009).

[6] S. Afach et al., Highly stable atomic vector magnetometer based on free spin precession, Opt. Express 23, 22108 (2015).
[7] A. Weis, G. Bison, N. Castagna, S. Cook, A. Hofer, M. Kasprzak, P. Knowles, and J.-L. Schenker, Mapping the cardiomagnetic field with 19 room temperature secondorder gradiometers, IFMBE Proc. 28, 58 (2010).

[8] V. Schultze, B. Schillig, R. IJsselsteijn, T. Scholtes, S. Woetzel, and R. Stolz, An optically pumped magnetometer working in the light-shift dispersed Mz mode, Sensors 17, 561 (2017).

[9] R. Wyllie, M. Kauer, R. T. Wakai, and T. G. Walker, Optical magnetometer array for fetal magnetocardiography, Opt. Lett. 37, 2247 (2012).

[10] H. Xia, A. Ben-Amar Baranga, D. Hoffman, and M. V. Romalis, Magnetoencephalography with an atomic magnetometer, Appl. Phys. Lett. 89, 211104 (2006).

[11] P. J. Broser, S. Knappe, D. Kajal, N. Noury, O. Alem, V. Shah, and C. Braun, Optically pumped magnetometers for magneto-myography to study the innervation of the hand, IEEE Trans. Neural Syst. Rehabil. Eng. 26, 2226 (2018).

[12] W. Hanle, Über magnetische Beeinflussung der Polarisation der Resonanzfluoreszenz, Zeitschrift für Physik 30, 93 (1924).

[13] H. Gilles, B. Cheron, and J. Hamel, Dispersive effects in optically pumped $(23 \mathrm{~s} 1)$ the atomic vapor measured by using a geometrical optics technique, Opt. Commun. 190, 179 (2001).

[14] W. Happer and A. C. Tam, Effect of rapid spin exchange on the magnetic-resonance spectrum of alkali vapors, Phys. Rev. A 16, 1877 (1977). 
[15] J. C. Allred, R. N. Lyman, T. W. Kornack, and M. V. Romalis, High-Sensitivity Atomic Magnetometer Unaffected by Spin-Exchange Relaxation, Phys. Rev. Lett. 89, 130801 (2002).

[16] F. Laloë, M. Leduc, and P. Minguzzi, Relations entre l'état angulaire d'une vapeur atomique soumise au pompage optique et ses propriétés d'absorption et de dispersion, J. de Physique 30, 277 (1969).

[17] J. Dupont-Roc, Détermination par des méthodes optiques des trois composantes d'un champ magnétique très faible, Revue de Physique Appliquée 5, 853 (1970).

[18] A. Korver, R. Wyllie, B. Lancor, and T. G. Walker, Suppression of Spin-Exchange Relaxation Using Pulsed Parametric Resonance, Phys. Rev. Lett. 111, 043002 (2013).

[19] O. Alem, R. Mhaskar, R. Jiménez-Martínez, D. Sheng, J. LeBlanc, L. Trahms, T. Sander, J. Kitching, and S. Knappe, Magnetic field imaging with microfabricated optically-pumped magnetometers, Opt. Express 25, 7849 (2017).

[20] E. Labyt, M. Corsi, W. Fourcault, A. P. Laloy, F. Bertrand, F. Lenouvel, G. Cauffet, M. L. Prado, F. Berger, and S. Morales, Magnetoencephalography with optically pumped ${ }^{4} \mathrm{He}$ magnetometers at ambient temperature, IEEE Trans. Med. Imaging 38, 90 (2018).

[21] Y. Shi, T. Scholtes, Z. D. Grujić, V. Lebedev, V. Dolgovskiy, and A. Weis, Quantitative study of optical pumping in the presence of spin-exchange relaxation, Phys. Rev. A 97, 013419 (2018).

[22] Y. Shi and A. Weis, Cesium alignment produced by pumping with unpolarized light, Eur. Phys. J. D 72, 73 (2018).

[23] E. Breschi and A. Weis, Ground-state Hanle effect based on atomic alignment, Phys. Rev. A 86, 053427 (2012).

[24] F. Beato, E. Belorizky, E. Labyt, M. Le Prado, and A. Palacios-Laloy, Theory of a $\mathrm{He} 4$ parametric-resonance magnetometer based on atomic alignment, Phys. Rev. A 98, 053431 (2018).

[25] F. D. Colegrove and P. A. Franken, Optical pumping of Helium in the 3s1 metastable state, Phys. Rev. 119, 680 (1960).

[26] G. Bevilacqua, E. Breschi, and A. Weis, Steady-state solutions for atomic multipole moments in an arbitrarily oriented static magnetic field, Phys. Rev. A 89, 033406 (2014).

[27] G. Colangelo, R. J. Sewell, N. Behbood, F. M. Ciurana, G. Triginer, and M. W. Mitchell, Quantum atom-light interfaces in the Gaussian description for spin-1 systems, New J. Phys. 15, 103007 (2013).

[28] S. I. Kanorsky, A. Weis, J. Wurster, and T. W. Hänsch, Quantitative investigation of the resonant nonlinear Faraday effect under conditions of optical hyperfine pumping, Phys. Rev. A 47, 1220 (1993).

[29] K. Blum, Density Matrix Theory and Applications (Springer Science \& Business Media, Berlin, 2012).

[30] S. Morales, M.-C. Corsi, W. Fourcault, F. Bertrand, G. Cauffet, C. Gobbo, F. Alcouffe, F. Lenouvel, M. L. Prado, F. Berger, G. Vanzetto, and E. Labyt, Magnetocardiography measurements with ${ }^{4} \mathrm{He}$ vector optically pumped magnetometers at room temperature, Phys. Med. Biol. 62, 7267 (2017).

[31] P. A. Franken and F. D. Colegrove, Alignment of Metastable Helium Atoms by Unpolarized Resonance Radiation, Phys. Rev. Lett. 1, 316 (1958).

[32] C. Cohen-Tannoudji, J. Dupont-Roc, S. Haroche, and F. Laloë, Diverses résonances de croisement de niveaux sur des atomes pompés optiquement en champ nul. I. Théorie, Revue de Physique Appliquée 5, 95 (1970).

[33] N. Tsukada, T. Yabuzaki, and T. Ogawa, Effect of transverse pumping in optical pumping, J. Phys. Soc. Jpn. 33, 698 (1972). 